J Am Soc Hypertens. 2014 July ; 8(7): 475-480. doi:10.1016/j.jash.2014.04.011.

\title{
GENETIC VARIATION IN CYP4A11 AND BLOOD PRESSURE RESPONSE TO MINERALOCORTICOID RECEPTOR ANTAGONISM OR ENAC INHIBITION: AN EXPLORATORY PILOT STUDY IN AFRICAN AMERICANS
}

\author{
Cheryl L. Laffer ${ }^{1}$, Fernando Elijovich ${ }^{1}$, George J. Eckert ${ }^{2}$, Wanzhu Tu${ }^{2,3}$, J. Howard Pratt ${ }^{2,4}$, \\ and Nancy J. Brown ${ }^{1}$ \\ ${ }^{1}$ the Department of Medicine, Vanderbilt University School of Medicine, Nashville, TN \\ ${ }^{2}$ the Department of Medicine, Indiana University School of Medicine, Indianapolis, IN \\ ${ }^{3}$ the Regenstrief Institute, Inc., Indianapolis, IN \\ ${ }^{4}$ the Richard L Roudebush VA Medical Center, Indianapolis, IN
}

\begin{abstract}
Background-An rs3890011 variant of CYP4A11, which is in linkage disequilibrium with the loss-of-function variant rs 1126742, is associated with hypertension in humans. In mice, Cyp $4 a$ deficiency results in salt-sensitive hypertension through activation of ENaC. We tested the hypothesis that the rs3890011 variant is associated with blood pressure response to drugs acting via the $\mathrm{ENaC}$ pathway.
\end{abstract}

\begin{abstract}
Methods and Results-African Americans with volume-dependent, resistant hypertension were randomized to treatment with placebo, spironolactone, amiloride, or combination. Blood pressure responses were analyzed by CYP4A11 genotypes. Rs3890011 (GG:GC:CC=20:35:28) and rs1126742 (TT:TC:CC $=45: 31: 7)$ were in linkage disequilibrium $\left(D^{\prime}=1, r=0.561\right)$. Expected small number of rs $1126742 \mathrm{CC}$ homozygotes precluded analysis of the effect of this genotype on treatment responses. Spironolactone reduced blood pressure in rs3890011 GG and GC individuals, but not in CC homozygotes ( $\mathrm{p}=0.002$ ), whereas amiloride reduced blood pressure similarly in all rs3890011 genotypes. The antihypertensive effects of spironolactone and amiloride were comparable in GG and GC participants, but only amiloride reduced pressure in CC homozygotes $(-6.3 \pm 7.3 /-3.2 \pm 4.0$ versus $+6.8 \pm 7.9 /+4.8 \pm 8.6 \mathrm{mmHg}, \mathrm{p}<0.01 /<0.05)$. The aldosterone response to spironolactone was also blunted in the $\mathrm{CC}$ genotype.
\end{abstract}

\footnotetext{
(C) 2014 American Society of Hypertension. Published by Elsevier Inc. All rights reserved.

Corresponding author: Cheryl L. Laffer, MD, PhD, Clinical Pharmacology 536RRB, Vanderbilt University Medical Center, Nashville, TN, 615-936-3420, Fax: 615-875-3297, cheryl.1.laffer@ vanderbilt.edu.

Conflict of Interest/Disclosures: None

Publisher's Disclaimer: This is a PDF file of an unedited manuscript that has been accepted for publication. As a service to our customers we are providing this early version of the manuscript. The manuscript will undergo copyediting, typesetting, and review of the resulting proof before it is published in its final citable form. Please note that during the production process errors may be discovered which could affect the content, and all legal disclaimers that apply to the journal pertain.
} 
Conclusions-In individuals homozygous for the CYP4A11 rs3890011 C allele, blood pressure is resistant to mineralocorticoid receptor antagonism, but sensitive to $\mathrm{ENaC}$ inhibition, consistent with $\mathrm{ENaC}$ activation. Studies in a larger population are needed to replicate these findings.

\section{Keywords}

hypertension; epithelial sodium channel; amiloride; spironolactone

\section{Introduction}

The cytochrome P450 $\omega$-hydroxylase CYP4A11 oxidizes arachidonic acid to 20hydroxyeicosatetraenoic acid (20-HETE), which causes both vasoconstriction and natriuresis. (1) In humans, a variant in intron 10 of CYP4A11 (rs3890011) with a relatively high minor allele frequency was associated with systolic blood pressure (SBP) in normotensive Chinese men ( $\mathrm{p}=0.05$ after controlling for age and body mass index), and a haplotype containing the $\mathrm{C}$ allele of rs3890011 was associated with hypertension in women. (2) A nearby loss-of-function variant in exon 11 of CYP4A11 (rs1126742, T8590C, or Phe434Ser), in linkage disequilibrium with rs 3890011 but with a much lower minor allele frequency (17.4\%), has been associated with hypertension in several populations. (3-6) In addition, this polymorphism has been associated with the sensitivity of blood pressure to salt intake in hypertensive individuals. (7)

In mice, genetic deficiency of the $\omega$-hydroxylase Cyp4a10 results in salt-sensitive hypertension but does not cause a change 20-HETE synthase activity. Hypertension results from increased sodium reabsorption due to constitutive activation of the epithelial sodium channel $(\mathrm{ENaC})$ mediated by a decrease in epoxygenase activity and renal synthesis of epoxyeicosatrienoic acids (EETs). (8) Treatment of Cyp4a10-/- mice with the ENaC antagonist amiloride normalizes blood pressure. This is analogous to the situation in Liddle syndrome, a Mendelian form of human hypertension in which mutations in either the $\beta$ or $\gamma$ subunit of ENaC result in gain-of-function and sodium retention that can be corrected by treatment with amiloride but not with spironolactone. $(9,10)$

The CYP4A11 variant rs1126742 encodes for an enzyme with reduced catalytic activity, (6) making it conceivable that the associated salt-sensitive hypertension is due to deficient 20HETE synthesis. In contrast, the mechanism for the association between the intronic variant rs3890011 and increased blood pressure is not known. We hypothesized that it may be associated with increased $\mathrm{ENaC}$ activity. If this were the case, the hypertension would be resistant to treatment with the mineralocorticoid receptor (MR) antagonist spironolactone but sensitive to the $\mathrm{ENaC}$ inhibitor amiloride. To test this hypothesis, we compared the blood pressure response to placebo, spironolactone, amiloride, and the combination of spironolactone and amiloride in an exploratory pilot study in 83 hypertensive African Americans who participated in a published randomized clinical trial, (11) and for whom DNA was available, according to the CYP4A11 genotype at rs3890011. 


\section{Methods}

The study protocol was approved by the Indiana University-Purdue University at Indianapolis institutional review board. All patients gave written informed consent.

Patients were eligible for enrollment if they were self-identified as black (defined as of African descent), between 18 and 75 years of age, and had a SBP >140 and $₫ 75 \mathrm{~mm} \mathrm{Hg}$ or a diastolic blood pressure (DBP) $>90$ and $₫ 05 \mathrm{~mm} \mathrm{Hg}$ while receiving any of the following - hydrochlorothiazide (minimum dose of $25 \mathrm{mg}$ ), furosemide (minimum dose of $40 \mathrm{mg}$ ), equivalent doses of similar diuretics and amlodipine 5 or $10 \mathrm{mg}$, or equivalent doses of a similar calcium channel blocker. (11) Any use of triamterene, an angiotensin-converting enzyme (ACE) inhibitor, or an angiotensin receptor blocker was discontinued for 1 month before the study. To restrict enrollment to patients with hypertension that was more volume dependent, patients were excluded if their plasma renin activity (PRA) exceeded $2.0 \mathrm{ng} / \mathrm{mL}$ per hour.

\section{Protocol}

Patients completed a randomized, placebo-controlled, double-blind, parallel-group trial that used a 2-by-2 factorial design, as previously described. (11) They were screened at least 3 weeks prior to randomization and eligible subjects were given 2 placebo capsules to take each morning for 3 weeks. They were then randomized to one of four treatment groups: amiloride (10 mg per day), spironolactone ( $25 \mathrm{mg}$ per day), the combination of both drugs, and placebo. Study medications were given as two identical appearing capsules. Blood pressure measurements and blood samples were obtained at baseline and at weeks 1, 3, 5, 7, and 9. Blood pressure was measured three times in the morning prior to study medication while patients were seated; the average of the last two readings was used in the analyses.

\section{Laboratory Analysis}

Serum electrolytes and creatinine were measured using a Vitros 950 instrument (Ortho Clinical Diagnostics). PRA was measured using a radioimmunoassay for angiotensin I (Clinical Assays GammaCoat kit); the intra-assay coefficient of variation (CV) was 4.6\%, and the interassay $\mathrm{CV}$ was $7.6 \%$. (11) Aldosterone was also measured by radioimmunoassay (Diagnostic Products Corporation), and the intra-assay and interassay CVs were 5.4\% and $13.1 \%$, respectively.

\section{Genetic Analysis}

Genotyping for SNP rs3890011 of CYP4A11 was performed by amplification and sequencing of a DNA segment covering exon 10 through exon 11, as previously described. (3) Genotyping for rs1126742 was also obtained to verify allele frequencies in our population.

\section{Statistical Analysis}

Data are presented as mean \pm standard deviation unless otherwise indicated. Primary end points were the changes from baseline in SBP and DBP at nine weeks of therapy. Secondary end points were changes in PRA and plasma aldosterone and in serum levels of potassium 
and creatinine. Change from baseline to 9 weeks was calculated for each study participant. Deviation from Hardy-Weinberg was calculated using chi square testing. The effect of genotype on response to treatment was tested using ANCOVA, in which the age at onset of hypertension, sex, and baseline were included as covariates. Change in potassium was also included as a covariate in an ANOVA assessing the effect of rs3890011 genotype on aldosterone concentrations. Plasma aldosterone concentrations and PRA were transformed using the natural $\log$ prior to analysis. A p value less than 0.05 was considered significant.

\section{Results}

The frequencies of both rs3890011 and rs1126742 were in Hardy-Weinberg equilibrium and their genotypes were in linkage disequilibrium $\left(D^{\prime}=1, \mathrm{r}=0.561, \mathrm{p}<0.001\right)$. The frequency of the minor allele for the loss-of-function variant at rs1126742 was $27.1 \%$, similar to the previously reported $30.0 \%$ for African Americans, (3) confirming our prediction that genotypic analyses of therapeutic responses would not be possible in a population the size of ours, owing to the low number of CC homozygous subjects (TT:TC:CC=45:31:7). The only significant difference related to rs 1126742 genotype was detected in all patients combined (four therapy groups). We observed a failure of aldosterone levels to increase in response to treatment in CC subjects (Figure 1), compared to TT and CT, who exhibited the expected increase in this hormone in response to MR or ENaC blockade. $(12,13)$

In contrast, the frequency of the minor allele for rs 3890011 was $54.8 \%$, higher in this group of African American subjects than that reported in Chinese (2) and in the 1000 Genomes database (41.5\%), (14) with a genotype distribution allowing for the testing of our hypothesis (GG:GC:CC=20:35:28).

Table 1 provides baseline characteristics for subjects according to rs3890011 genotype. There was no difference in blood pressure, the number of anti-hypertensive medications, or any other baseline variable among genotypes. There was also no difference in the classes of anti-hypertensive medications used among genotype groups randomized to spironolactone or not.

Figure 2 shows that placebo-induced changes in blood pressure over the period of study were not different among the three rs 3890011 genotypes (panel A). There was no reduction in SBP and DBP in response to spironolactone monotherapy in the $5 \mathrm{CC}$ homozygous subjects, significantly different from the reductions observed in the 7 GG and 8 GC (panel B). In contrast, amiloride monotherapy reduced SBP and DBP similarly in all CYP4A11 rs3890011 genotypes (GG:GC:CC=6:8:6, panel C). In a sensitivity analysis, there was a significant association between CYP4A11 rs3890011 genotype (GG:GC:CC=12:15:13) and blood pressure response to any spironolactone-containing treatment (Figure 3) but not to any amiloride-containing therapy ( $>0.15$, not shown). Finally, in all subjects carrying at least one rs3890011 $\mathrm{G}$ allele $(\mathrm{GG}+\mathrm{GC})$ amiloride reduced SBP and DBP $(-10.6 \pm 8.2 /-5.9 \pm 6.4$ $\mathrm{mmHg})$ to the same extent as spironolactone $(-9.8 \pm 9.4 /-6.3 \pm 6.5 \mathrm{mmHg}), \mathrm{p}=0.41 / 0.43$. In contrast, in CC homozygous subjects amiloride reduced SBP and DBP $(-6.3 \pm 7.3 /-3.2 \pm 4.0$ $\mathrm{mmHg})$ to a greater extent than spironolactone did $(+6.8 \pm 7.9 /+4.8 \pm 8.6), \mathrm{p}<0.01 /<0.05$. The increase in SBP of CC with spironolactone monotherapy was significant $(\mathrm{p}<0.03)$ but not 
significantly different from that sustained by all subjects given placebo during the trial (3.6 \pm 11.3$), p=0.28$. There was no effect of gender or BMI on response to spironolactone or amiloride, alone or in combination.

Aldosterone responses to therapy followed a similar pattern. In subjects carrying at least one G allele (GG+GC), amiloride increased aldosterone from $452 \pm 58$ to $784 \pm 97 \mathrm{pmol} / \mathrm{L}$ and spironolactone increased aldosterone from $352 \pm 53$ to $596 \pm 91 \mathrm{pmol} / \mathrm{L}$ (drug comparison not significant, adjusted marginal means after controlling for gender and change in potassium). In contrast, in CC homozygous subjects, amiloride increased aldosterone from $585 \pm 89$ to $989 \pm 144 \mathrm{pmol} / \mathrm{L}$, whereas spironolactone did not affect aldosterone (from $446 \pm 91$ to $449 \pm 147 \mathrm{pmol} / \mathrm{L}, \mathrm{p}=0.038$ for drug comparison). There were no other significant relationships between CYP4A11 rs3890011 and the effect of either spironolactone or amiloride on heart rate, PRA, plasma sodium, plasma potassium, or creatinine.

\section{Discussion}

Gain-of-function mutations in $\mathrm{ENaC}$ such as occur in Liddle syndrome result in saltsensitive hypertension that is resistant to $\mathrm{MR}$ antagonists but responds to the $\mathrm{ENaC}$ inhibitor amiloride. $(9,10)$ In rodent models, decreased expression of Cyp $4 a$ also results in increased $\mathrm{ENaC}$ activity and salt-sensitive hypertension that is responsive to amiloride. $(8,15) \mathrm{We}$ report for the first time in this exploratory pilot study that African Americans with resistant, volume-dependent hypertension who are homozygous for the $\mathrm{C}$ allele at rs3890011 of CYP4A11, an allele previously associated with blood pressure (2), are resistant to the blood pressure lowering effects of spironolactone but respond to amiloride.

Several lines of evidence in rodents suggest that CYP4A modulates ENaC function. First, Nakagawa et al reported that genetic deficiency of CYP4a10 results in constitutive activity of $\mathrm{ENaC}$ and salt-sensitive hypertension that is responsive to amiloride. (8) This is thought to result from decreased formation of EETs that act as endogenous inhibitors of ENaC. In the Dahl salt-sensitive rat, CYP4A genotype segregates with salt-sensitive hypertension. (16) Decreased CYP4A hydroxylase activity results in salt-sensitive hypertension in this model, whereas induction of CYP4A increases pressure natriuresis. More recently, Kakizoe et al have reported that $\beta$ and $\gamma \mathrm{ENaC}$ subunit mRNA and protein are overexpressed in Dahl saltsensitive rats. (15) They further reported that 4-week treatment with amiloride reduced blood pressure and renal injury whereas treatment with eplerenone did not.

A common intronic variant of CYP4A11, rs3890011, is associated with blood pressure in the Chinese. (2) We studied the association between this variant and the blood pressure responses to amiloride and spironolactone in our population. The rs 3890011 locus is 247 base pairs away and in linkage disequilibrium with rs1126742, a rarer coding variant of CYP4A11. The latter variant in exon 11 encodes for a thymidine-to-cytosine substitution at base pair 8590 that results in a Phe-to-Ser substitution at amino acid 434 and loss of function of the product enzyme. (3) The low frequency of the minor allele of rs 1126742 precludes genotypic analyses in populations the size of ours, owing to the small number of homozygous CC subjects. Therefore, we did not study the relationship between this genotype and blood pressure responses to therapy in our small clinical trial. We were only 
able to document that the aldosterone response to treatment was diminished in rs 1126742 CC homozygous subjects.

Whether there is a functional consequence of the rs 3890011 intronic variant is not currently known but the much larger frequency of its minor allele allowed us to examine the relationship between this CYP4A11 polymorphism and the blood pressure response to spironolactone and amiloride in African Americans with resistant hypertension and suppressed PRA. The prevalence of salt-sensitive or volume-dependent hypertension is increased among African Americans. (17) Genetic variants in $\mathrm{ENaC}$ that have been associated with an increased urinary aldosterone/potassium ratio, an index of $\mathrm{ENaC}$ activity, are more common in African Americans but have not been universally associated with hypertension. $(18,19)$ As previously published in this study population of African Americans with volume-dependent resistant hypertension, amiloride alone has a greater effect on blood pressure than does spironolactone alone. (11) Whereas it is possible that this relates to the doses of spironolactone and amiloride used, our data suggest that this is also attributable to a diminished response to spironolactone in subjects homozygous for the CYP4A11 rs3890011 $\mathrm{C}$ allele.

The loss-of-function CYP4A11 rs1126742 C allele is more common in African Americans than in other ancestral groups (3) and we found the CYP4A11 rs3890011 C allele to be more common in our African American study population than previously reported in Chinese. (2) Whether the findings of this study will hold up in individuals with resistant hypertension from other racial groups remains to be studied.

A limitation of our study is the small number of subjects, although the sensitivity analysis in which we combined subjects in spironolactone treatment groups (Figure 3) supports the conclusions. Also, baseline PRA and aldosterone concentrations were not significantly lower in rs3890011 CC homozygotes, as one would expect if $\mathrm{ENaC}$ activity were increased. The exclusion of subjects with a PRA greater than $2.0 \mathrm{ng} / \mathrm{mL}$ per hour and the use of concomitant medications that affect PRA (e.g., diuretics and $\beta$-blockers) may have confounded any relationship between CYP4A11 genotype and baseline PRA. Williams et al previously reported the relationship between the CYP4A11 rs1126742 genotype and saltsensitivity of blood pressure in untreated subjects. (7) In that study, although there was no significant relationship between genotype and PRA or aldosterone overall, there was a significant relationship between CYP4A11 genotype and aldosterone in African Americans during low salt $(16.5 \pm 9.5,14.9 \pm 7.2$, and $7.9 \pm 3.4 \mathrm{ng} / \mathrm{dL}$, in $36 \mathrm{TT}, 33 \mathrm{CT}, 5 \mathrm{CC}$, respectively, $\mathrm{p}=0.03$, unpublished). There was no relationship between CYP4A11 genotype and PRA in African Americans in that study (unpublished).

In summary, inappropriate activation of $\mathrm{ENaC}$ has increasingly been recognized as a feature of resistant hypertension. (20) The MR antagonists spironolactone and eplerenone are often effective in reducing blood pressure in patients with resistant hypertension. $(21,22)$ Not all patients respond to MR antagonism, however, and in these patients amiloride may be more effective. (11) Genetic variants in CYP4A11 have been associated with hypertension and with salt-sensitive hypertension in particular. (2-7) The present data suggest that genetic 
variation in CYP4A11 might be used to predict individuals who should be treated with a direct $\mathrm{ENaC}$ inhibitor. Additional studies are needed to replicate these findings.

\section{Acknowledgments}

Source of Funding: This work was supported by National Institutes of Health grants HL006730, HL003579, RR000750, and DK038226.

The authors wish to thank Elliott P. Dawson for assistance with genotyping.

\section{References}

1. Zordoky BN, El-Kadi AO. Effect of cytochrome P450 polymorphism on arachidonic acid metabolism and their impact on cardiovascular diseases. Pharmacol Ther. 2010; 125:446-63. [PubMed: 20093140]

2. Zhang R, Lu J, Hu C, Wang C, Yu W, Ma X, et al. A common polymorphism of CYP4A11 is associated with blood pressure in a Chinese population. Hypertens Res. 2011; 34:645-8. [PubMed: 21326303]

3. Gainer JV, Bellamine A, Dawson EP, Womble KE, Grant SW, Wang Y, et al. Functional variant of CYP4A11 20-hydroxyeicosatetraenoic acid synthase is associated with essential hypertension. Circulation. 2005; 111:63-9. [PubMed: 15611369]

4. Mayer B, Lieb W, Gotz A, Konig IR, Aherrahrou Z, Thiemig A, et al. Association of the T8590C polymorphism of CYP4A11 with hypertension in the MONICA Augsburg echocardiographic substudy. Hypertension. 2005; 46:766-71. [PubMed: 16144986]

5. Hermann M, Hellermann JP, Quitzau K, Hoffmann MM, Gasser T, Meinertz T, et al. CYP4A11 polymorphism correlates with coronary endothelial dysfunction in patients with coronary artery disease-The ENCORE Trials. Atherosclerosis. 2009; 207:476-9. [PubMed: 19615687]

6. Gainer JV, Lipkowitz MS, Yu C, Waterman MR, Dawson EP, Capdevila JH, et al. Association of a CYP4A11 variant and blood pressure in black men. J Am Soc Nephrol. 2008; 19:1606-12. [PubMed: 18385420]

7. Williams JS, Hopkins PN, Jeunemaitre X, Brown NJ. CYP4A11 T8590C polymorphism, saltsensitive hypertension, and renal blood flow. J Hypertens. 2011; 29:1913-8. [PubMed: 21873888]

8. Nakagawa K, Holla VR, Wei Y, Wang WH, Gatica A, Wei S, et al. Salt-sensitive hypertension is associated with dysfunctional Cyp4a10 gene and kidney epithelial sodium channel. J Clin Invest. 2006; 116:1696-702. [PubMed: 16691295]

9. Hansson JH, Schild L, Lu Y, Wilson TA, Gautschi I, Shimkets R, et al. A de novo missense mutation of the beta subunit of the epithelial sodium channel causes hypertension and Liddle syndrome, identifying a proline-rich segment critical for regulation of channel activity. Proc Natl Acad Sci U S A. 1995; 92:11495-9. [PubMed: 8524790]

10. Hansson JH, Nelson-Williams C, Suzuki H, Schild L, Shimkets R, Lu Y, et al. Hypertension caused by a truncated epithelial sodium channel gamma subunit: genetic heterogeneity of Liddle syndrome. Nat Genet. 1995; 11:76-82. [PubMed: 7550319]

11. Saha C, Eckert GJ, Ambrosius WT, Chun TY, Wagner MA, Zhao Q, et al. Improvement in blood pressure with inhibition of the epithelial sodium channel in blacks with hypertension. Hypertension. 2005; 46:481-7. [PubMed: 16116042]

12. Dluhy RG, Axelrod L, Underwood RH, Williams GH. Studies of the control of plasma aldosterone concentration in normal man. II. Effect of dietary potassium and acute potassium infusion. J Clin Invest. 1972; 51:1950-7. [PubMed: 5054456]

13. Pratt JH, Eckert GJ, Newman S, Ambrosius WT. Blood pressure responses to small doses of amiloride and spironolactone in normotensive subjects. Hypertension. 2001; 38:1124-9. [PubMed: 11711509]

14. The 1000 Genomes Project Consortium. An integrated map of genetic variation from 1,092 human genomes. Nature. 2012; 491:56-65. [PubMed: 23128226] 
15. Kakizoe Y, Kitamura K, Ko T, Wakida N, Maekawa A, Miyoshi T, et al. Aberrant ENaC activation in Dahl salt-sensitive rats. J Hypertens. 2009; 27:1679-89. [PubMed: 19458538]

16. Williams JM, Sarkis A, Hoagland KM, Fredrich K, Ryan RP, Moreno C, et al. Transfer of the CYP4A region of chromosome 5 from Lewis to Dahl S rats attenuates renal injury. Am J Physiol Renal Physiol. 2008; 295:F1764-77. [PubMed: 18842817]

17. Weinberger MH. Salt sensitivity of blood pressure in humans. Hypertension. 1996; 27:481-90. [PubMed: 8613190]

18. Ambrosius WT, Bloem LJ, Zhou L, Rebhun JF, Snyder PM, Wagner MA, et al. Genetic variants in the epithelial sodium channel in relation to aldosterone and potassium excretion and risk for hypertension. Hypertension. 1999; 34:631-7. [PubMed: 10523338]

19. Baker EH, Dong YB, Sagnella GA, Rothwell M, Onipinla AK, Markandu ND, et al. Association of hypertension with T594M mutation in beta subunit of epithelial sodium channels in black people resident in London. Lancet. 1998; 351:1388-92. [PubMed: 9593408]

20. Bubien JK. Epithelial Na+ channel (ENaC), hormones, and hypertension. J Biol Chem. 2010; 285:23527-31. [PubMed: 20460373]

21. Calhoun DA, Nishizaka MK, Zaman MA, Thakkar RB, Weissmann P. Hyperaldosteronism among black and white subjects with resistant hypertension. Hypertension. 2002; 40:892-6. [PubMed: 12468575]

22. Weinberger MH, White WB, Ruilope LM, MacDonald TM, Davidson RC, Roniker B, et al. Effects of eplerenone versus losartan in patients with low-renin hypertension. Am Heart J. 2005; 150:426-33. [PubMed: 16169319] 


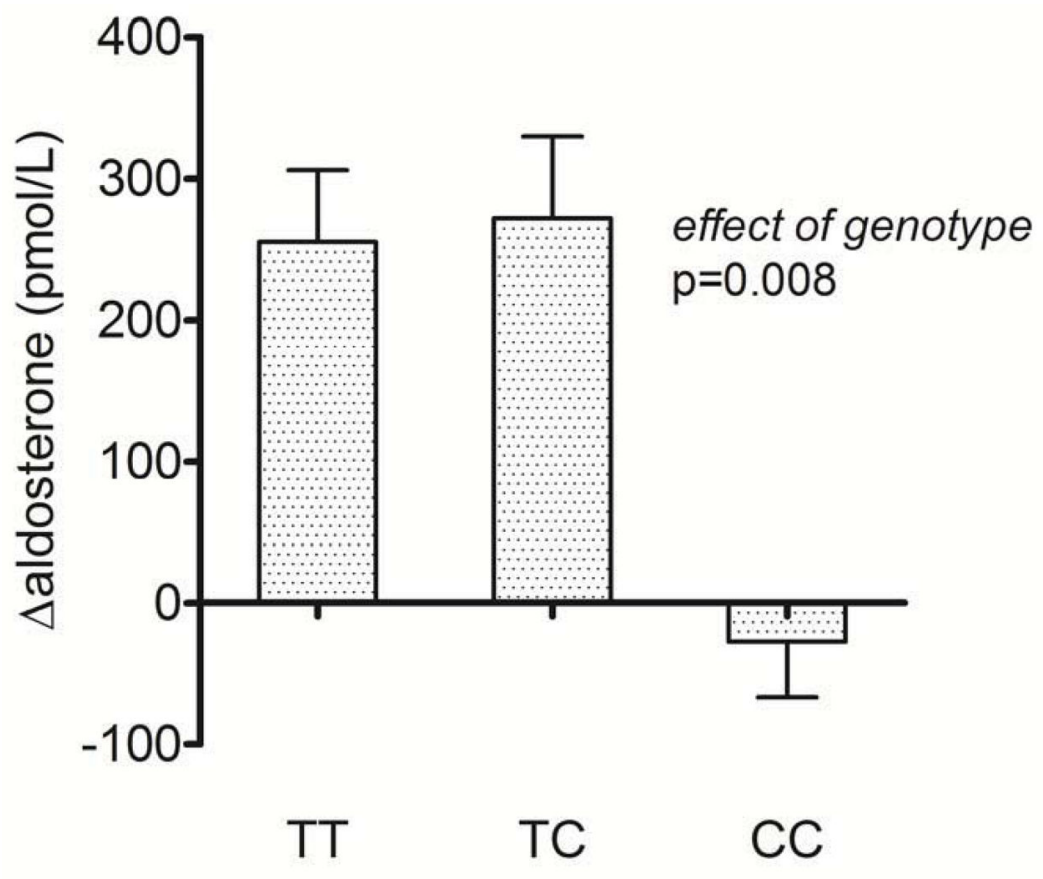

rs1126742 genotype

Figure 1.

Relationship between CYP4A11 rs1126742 (T8590C) genotype and aldosterone response to any therapy including placebo, spironolactone, amiloride or combination amiloride and spironolactone. Data are presented as means \pm standard error. 

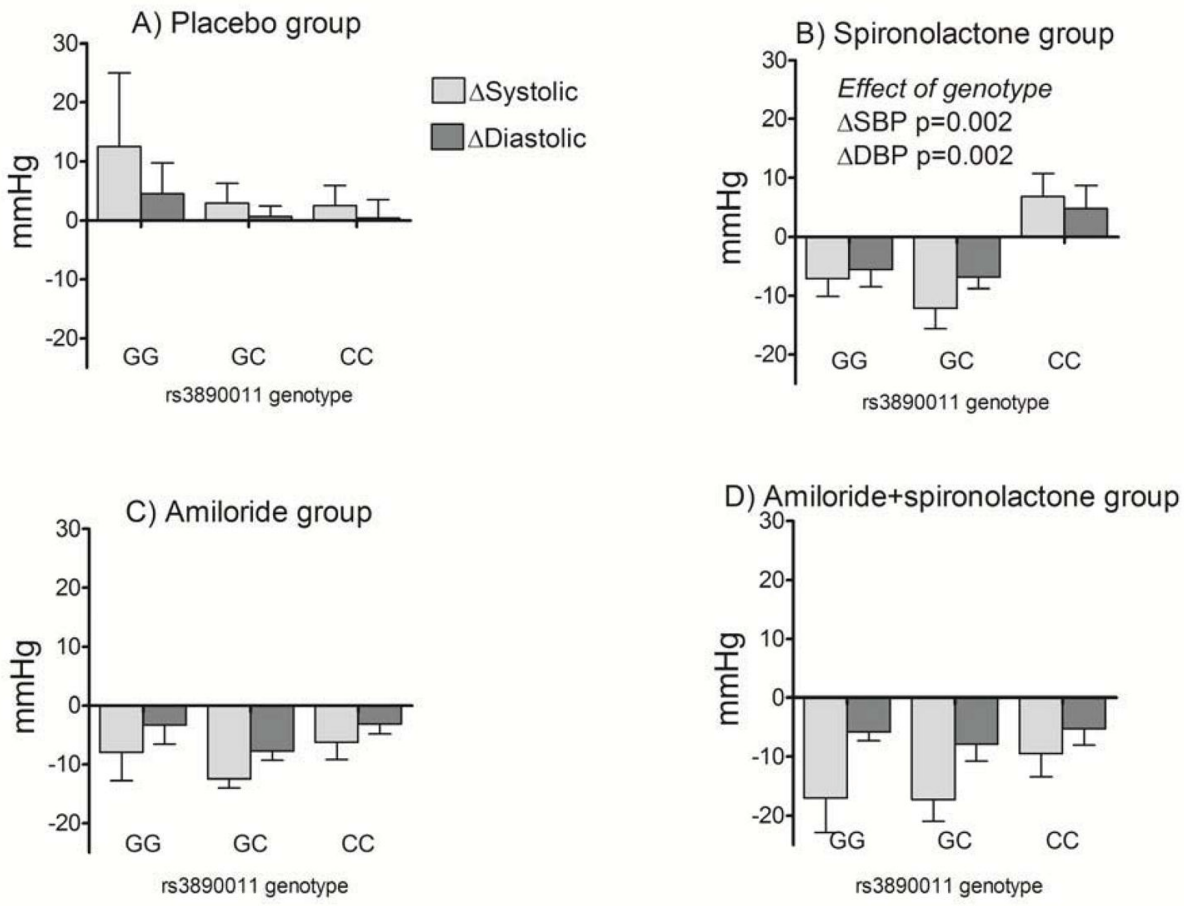

Figure 2.

Relationship between $C Y P 4 A 11$ rs3890011 genotype and blood pressure response after nine weeks of treatment with (A) placebo, (B) spironolactone, (C) amiloride or (D) combination amiloride and spironolactone. Data are presented as means \pm standard error. 


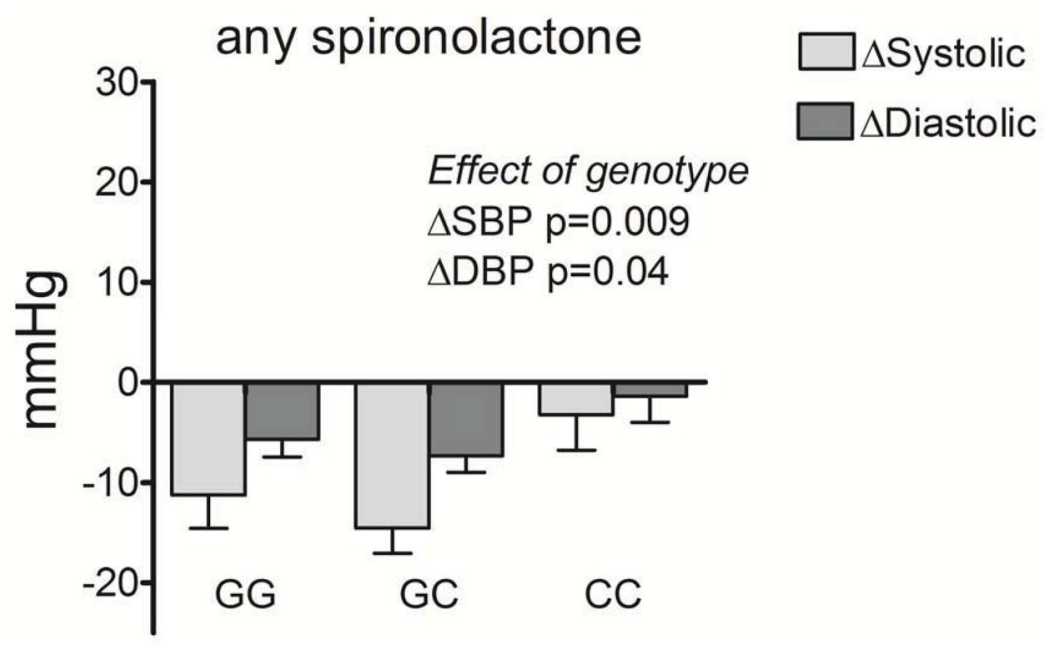

Figure 3.

Relationship between CYP4A11 rs3890011 genotype and blood pressure response to spironolactone in the presence or absence of amiloride (any spironolactone). Data are presented as means \pm standard error. 
Table 1

Baseline subject characteristics by CYP4A11 rs3890011 genotype

\begin{tabular}{|c|c|c|c|}
\hline CYP4A11 rs3890011 Genotype & GG $(\mathbf{N}=20)$ & GC $(N=35)$ & $\mathrm{CC}(\mathrm{N}=28)$ \\
\hline Gender, M:F & $12: 8$ & $16: 19$ & $16: 12$ \\
\hline Age, years & $49.0 \pm 9.0$ & $44.5 \pm 9.9$ & $47.9 \pm 8.7$ \\
\hline Body Mass Index, $\mathrm{kg} / \mathrm{M}^{2}$ & $32.8 \pm 4.9$ & $34.3 \pm 7.4$ & $35.4 \pm 10.0$ \\
\hline Duration of Hypertension, years & $13.2 \pm 8.2$ & $8.8 \pm 7.9$ & $12.2 \pm 8.1$ \\
\hline Number of Anti-Hypertensives & $2.35 \pm 0.67$ & $2.29 \pm 0.46$ & $2.21 \pm 0.50$ \\
\hline \multicolumn{4}{|l|}{ Class of Anti-Hypertensives, N (\%) } \\
\hline Thiazide diuretic & $19(95.0 \%)$ & $31(88.6 \%)$ & $25(89.3 \%)$ \\
\hline Loop diuretic & $1(5.0 \%)$ & $4(11.4 \%)$ & $3(10.7 \%)$ \\
\hline Amlodipine or other dihydropyridine & $19(95.0 \%)$ & $33(94.3 \%)$ & $28(100.0 \%)$ \\
\hline CCB other & $1(5.0 \%)$ & $1(2.9 \%)$ & 0 \\
\hline$\beta$-blocker & $6(30.0 \%)$ & $10(28.6 \%)$ & $6(21.4 \%)$ \\
\hline Alpha-2 agonist & $2(10 \%)$ & 0 & 0 \\
\hline Minoxidil & 0 & 0 & $1(3.8 \%)$ \\
\hline Alpha-1 antagonist & 0 & $1(2.8 \%)$ & $1(3.8 \%)$ \\
\hline Systolic Blood Pressure (mmHg) & $145.8 \pm 12.1$ & $139.9 \pm 10.5$ & $140.9 \pm 12.8$ \\
\hline Diastolic Blood Pressure (mmHg) & $91.5 \pm 7.8$ & $92.1 \pm 6.0$ & $89.4 \pm 8.9$ \\
\hline Heart Rate (bpm) & $79.4 \pm 9.2$ & $75.5 \pm 10.3$ & $78.5 \pm 8.8$ \\
\hline Glucose $(\mathbf{m g} / \mathbf{d L})$ & $123.6 \pm 39.0$ & $105.7 \pm 30.8$ & $117.4 \pm 46.4$ \\
\hline Serum sodium $(\mathbf{m m o l} / \mathbf{L})$ & $141.8 \pm 2.0$ & $142.1 \pm 1.9$ & $141.6 \pm 2.4$ \\
\hline Serum potassium $(\mathbf{m m o l} / \mathbf{L})$ & $3.6 \pm 0.4$ & $3.6 \pm 0.4$ & $3.7 \pm 0.3$ \\
\hline Serum creatinine $(\mathrm{mmol} / \mathrm{L})$ & $90.2 \pm 15.0$ & $91.9 \pm 18.6$ & $92.8 \pm 24.8$ \\
\hline Plasma Renin Activity (ng/mL/min) & $0.89 \pm 1.90$ & $0.77 \pm 1.87$ & $0.64 \pm 0.96$ \\
\hline Aldosterone (pmol/L) & $401.7 \pm 232.7$ & $407.2 \pm 193.9$ & $423.8 \pm 205.0$ \\
\hline
\end{tabular}

